\title{
Floral traits are associated with the quality but not quantity of heterospecific stigmatic pollen loads
}

\author{
Manon A. Peuker, Hannah Burger, Sabrina Krausch, Ulrich Neumüller, Manfred Ayasse and Jonas Kuppler *i)
}

\begin{abstract}
Background: In flowering communities, plant species commonly share pollinators and therefore plant individuals receive heterospecific pollen (HP). However, the patterns of HP transfers can deviate from patterns of plant-pollinator visitations. Although flower-visitor interactions are known to be mediated by floral traits, e.g. floral size or nectar tube depth, the explanatory power of these traits for HP transfer patterns remains elusive. Here, we have explored pollen transfer patterns at three sites in Southern Germany on three dates (early, mid and late summer). At the plant level, we tested whether flower abundance and floral traits are correlated with HP reception and donation. At the community level, we determined whether flower and bee diversity are correlated with network modularity and whether floral traits explain the module affiliation of plant species. We collected the stigmas of flowering plant species, analysed HP and conspecific pollen (CP) loads and measured floral traits, flower and bee diversity.

Results: Our results show that the degree and intensity of HP reception or donation at the plant level do not correlate with floral traits, whereas at the community level, the module affiliation of who is sharing pollen with whom is well-explained by floral traits. Additionally, variation in network modularity between communities is better explained by plant diversity and abundance than by bee diversity and abundance.

Conclusions: Overall, our results indicate that floral traits that are known to mediate flower-visitor interactions can improve our understanding of qualitative HP transfer but only provide limited information about the quantity of HP transfer, which more probably depends on other floral traits, flower-visitor identity or community properties.
\end{abstract}

Keywords: Pollination, Community, Pollen transfer, Functional trait

\section{Background}

In flowering communities, heterospecific pollen (HP) transfer is common and can result in negative consequences for plant reproductive success such as reduced pollen tube growth and seed production [1-6]. HP transfer mainly occurs when various plant species share the same flower visitors [7], although wind-dispersed pollen and other random events may also play a role $[3,8]$. During foraging, insects often visit and collect pollen

*Correspondence: jonas.kuppler@mail.de

Institute of Evolutionary Ecology and Conservation Genomics, Ulm

University, Albert-Einstein-Allee 11, 89081 Ulm, Germany from flowers from multiple plant species and may consequently transfer con- and heterospecific pollen (CP / HP) to a stigma [9]. However, the CP-/HP loads on stigmas often do not match flower-visitation patterns $[7,10,11]$.

Within a community, flower-visitor interactions can be summarized in networks depicting multi-species interactions [12, 13]. Such networks can also depict interactions based on pollen transfer instead of flower visitors. If flower-visiting insects are the main vector for pollen transport between plant individuals, factors that mediate flower-visitor interactions might also mediate pollen transfer patterns. Common mediators are: flower 
abundance or diversity, flower visitor diversity or floral traits $[14,15]$.

Abundant plant species often receive more visits from a more diverse insect community $[14,16]$. Therefore, individuals of abundant plant species might receive more $\mathrm{CP} /$ HP or their pollen might be distributed across a larger number of diverse plant species. Whereas this relationship between plant abundance and pollen transfer has been observed in nature [17], floral traits or pollination mode can contribute equally or more to pollen transfers [4].

In diverse plant and animal communities, increasing competition between flower visitors can lead to increasing specialization and resource partitioning $[15,16,18]$. As a consequence, flowering communities with a high species richness are often separated into different subnetworks of interacting species, i.e. are more modular $[19,20]$. Within one module, plant species share the same flower visitors thereby increasing the chance of exchanging pollen with each other. Thus, in pollen transfer networks, modularity may increase with plant and flower-visitor diversity.

Floral traits, such as morphology, colour or scent, have been shown to mediate flower-visitor interactions [15, 21-24]. During foraging, flower visitors of different functional groups or species often prefer plant species with specific floral phenotypes [25]. Thus, plant species displaying the expression of similar floral traits might have a high likelihood of receiving pollen from or of donating pollen to each other. For example, the radius of the floral tube can partially explain the modularity in pollen transfer networks in terms of the presence or absence of interactions, i.e. its quality [7]. However, patterns for the intensity or quantity of pollen transfer, i.e. the amount of CP / HP pollen received or donated, are not well explained by floral traits $[7,26]$.

Associations between floral traits, HP reception or donation and network properties are variable and even contradictory [3, 26, 27]. In general, stigma size, flower symmetry and floral size increase the likelihood and intensity of HP reception [3, 27, 28]. Short styles have been suggested to increase or decrease HP susceptibility $[1,4,27]$. The picture is similar for network properties. The in-degree, i.e. the number of plant species that receive pollen, is positively correlated with style length $[26,27]$. In contrast, the number of plant species that donate pollen, i.e. out-degree, is either not correlated [27] or the correlation patterns differ [26]. However, correlation studies concerning associations between pollen transfer, floral traits and community patterns are scarce [26].

The aim of our study has been to determine the way that floral traits, flower and bee abundance and diversity are associated with patterns of pollen reception and donation in natural communities. We have investigated whether these predictors are correlated with properties of pollen transfer networks. In order to analyse HP and CP loads, we have collected the stigmas of flowering plant species at three sites in Southern Germany on three different dates (early summer, mid-summer and late summer). Further, we have measured morphological floral traits (stamen length, inflorescence diameter, nectar tube width, nectar tube depth, floral display size and style length) and flower and bee abundance and diversity. Specifically, we have asked the following four questions: (I) Are pollen receipt and donation (in- and out-degree) and its intensity (weighted in- and out-degree) correlated with specific floral trait expression or flower abundance? (II) Is the modularity of pollen transfer networks linked to community properties such as bee or plant species richness? (III) Can floral trait expressions explain species module affiliation in pollen transfer networks? (IV) Do plant species with similar floral trait expression receive a similar HP percentage?

\section{Results}

In total, 116,954 pollen grains $(110,365 \mathrm{CP}$ and 6,589 $\mathrm{HP}$ ) on 1,117 stigmas resulting in 347 interspecific pollen transfers (IPT) were sampled across all communities (Table 1). Between communities, the number of IPTs ranged from 8 to 91 . The number of HP grains per stigma was generally small compared with the number of pollen grains (mean \%-CP load per stigma \pm sd, $86.98 \pm 26.75 \%$, range $0-100 \%)$. In general, CP was present on $~ 99 \%$ $(n=105)$, HP on $\sim 42 \%(n=470)$ and no pollen only on $\sim 0.05 \%(n=55)$ of all stigmas. The number of average pollen grains per stigma ranged from 1.3 to 748.8 . The maximum number of HP grains found on one stigma was 386 (Campanula rotundifolia) and the maximum number of pollen grains of different plant species identified on one stigma was 6 (Campanula rotundifolia, Centaurea jacea, Asparagaceae sp.2).

The frequency distribution of in- and out-degree was right-skewed and variable in all IPT networks (Fig. 1). For the in-degree, several zeros occurred attributable to HP grains of plants that were currently not flowering on our plots but that might have been flowering in the surroundings or some time before the sampling was conducted. In all IPT networks, most of the species (range: 75 - 100\%) received HP grains from at least one species. Approximately $46 \%$ of these species received pollen from one or two other species, whereas some species received pollen from a large number of species. For example, the highest in-degree $(=13)$ was found for Campanula rotundifolia (Eselsburger Tal, mid-summer), whereas an in-degree of 10 was recorded for four other species: Agrimonia 
Table 1 Descriptive results of sampling and pollen transfer within all nine communities

\begin{tabular}{|c|c|c|c|c|c|c|c|c|c|}
\hline & EB I & EB II & EB III & HT I & HT II & HT III & RB I & RB II & RB III \\
\hline No. Species* & $4(4)$ & $17(20)$ & $7(9)$ & $7(9)$ & $16(19)$ & $17(19)$ & $8(9)$ & $16(20)$ & $22(24)$ \\
\hline Total no. stigmas & 48 & 160 & 46 & 72 & 178 & 156 & 71 & 162 & 224 \\
\hline Total no. pollen grains & 11,030 & 21,564 & 8373 & 8409 & 13,956 & 11,279 & 9446 & 9324 & 23,573 \\
\hline Total no. CP grains & 10,472 & 20,712 & 8187 & 8056 & 12,211 & 10,883 & 9280 & 8668 & 21,896 \\
\hline Total no. HP grains & 558 & 852 & 186 & 353 & 1745 & 396 & 166 & 656 & 1677 \\
\hline Total no. links ${ }^{+}$ & 8 & 48 & 14 & 12 & 57 & 57 & 11 & 49 & 91 \\
\hline \%Donor & 25 & 43.75 & 28.57 & 42.86 & 20 & 47.06 & 37.5 & 37.5 & 36.36 \\
\hline \%Receptor & 50 & 56.25 & 57.14 & 57.14 & 73.33 & 47.06 & 25 & 56.25 & 54.54 \\
\hline \%Donor-Receptor & 25 & 0 & 14.29 & 0 & 6.77 & 5.88 & 37.5 & 6.25 & 9.1 \\
\hline Corr. In- and out-degree & $-0.30^{\mathrm{NS}}$ & $-0.23^{\mathrm{NS}}$ & $0.62^{\mathrm{NS}}$ & $-0.11^{\mathrm{NS}}$ & $-0.23^{\mathrm{NS}}$ & $-0.09^{N S}$ & $-0.53^{\mathrm{NS}}$ & $-10.19^{N S}$ & $-0.10^{\mathrm{NS}}$ \\
\hline Modularity & 0 & 0.38 & 0.1 & 0.25 & 0.47 & 0.48 & 0.31 & 0.56 & 0.47 \\
\hline
\end{tabular}

$\mathrm{EB}=$ Eselburger Tal, $\mathrm{HT}=$ Hirschtal, $\mathrm{RB}=$ Reichenbach. Roman numerals show sampling date: $\mathrm{I}=$ early summer, $\|=$ mid-summer, $I I I=$ late summer. No. species $=$ Total number of species, Total no. stigmas $=$ Total number of collected stigma, Total no. pollen grains $=$ Total number of pollen grains found on all stigmas, Total no. CP grains $=$ Total number of CP grains found on all stigmas, Total no. HP grains =Total number of HP grains found on all stigmas, Total no. links $=$ Total number of links found in the plant-plant pollen transfer network, \%Donor=Percentage hub-donor species in the network, i.e. species that donated HP to more species than from which they received $\mathrm{HP}$, \%Receptor = Percentage receptor species in the network, i.e. species that received HP from more species than to which they donate HP(??), $\%$ Donor-Receptor $=$ Percentage of species that donated and received pollen from the same number of species. Corr. In- and out-degree $=$ Pearson product correlation $r$ for in- and out-degree for each species within a community; ns non-significant. Modularity=Modularity for HP transfer network calculated using the optimization algorithm of Blondel et al. [43]

${ }^{*}$ No. species $=$ Number of species from which stigmas were collected (Number of flowering plant species. Some species were under protection or only present with $<3$ individuals and were excluded)

+ Total no. links = in each network, including links resulting from species that were not on our plots

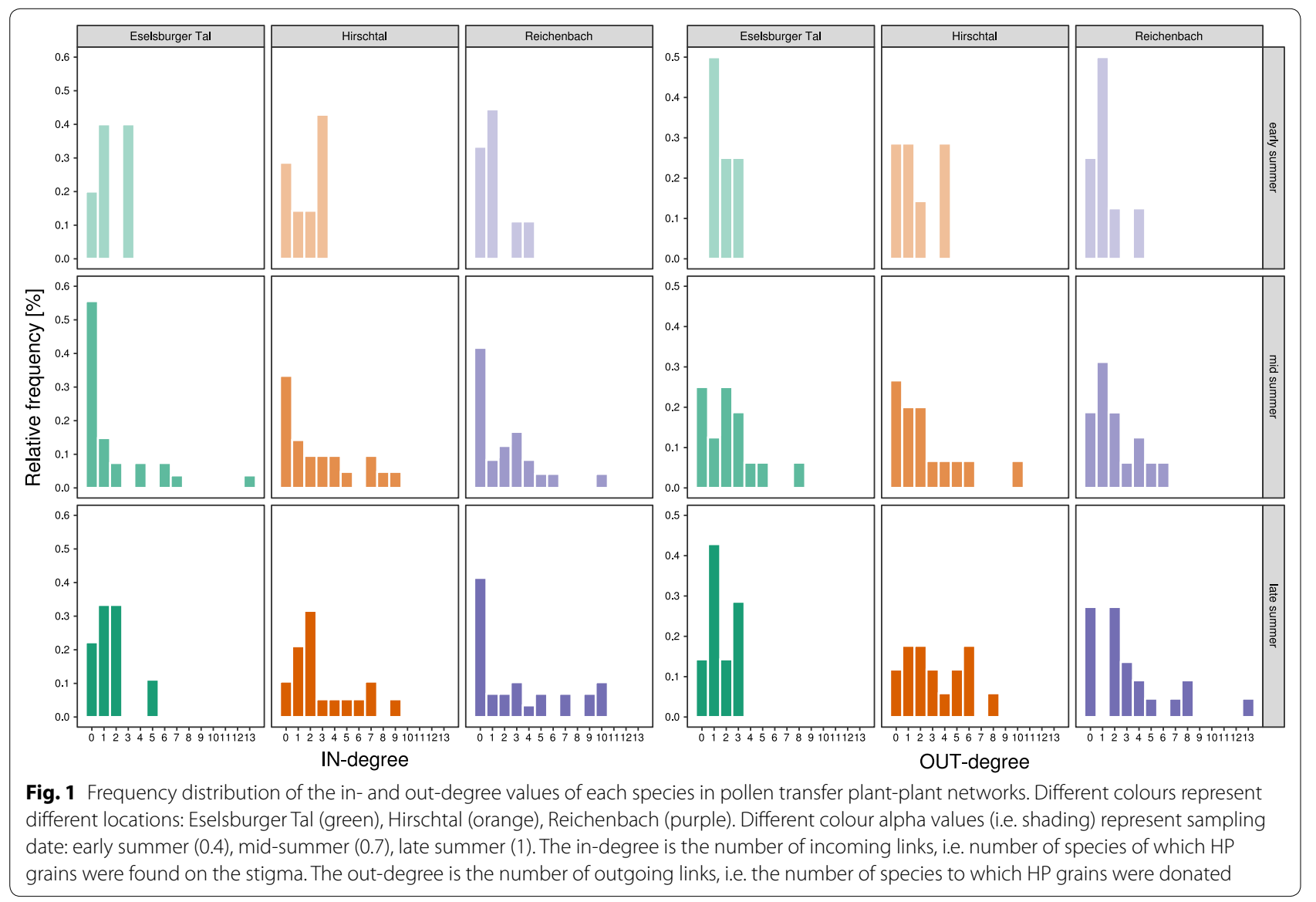


eupatoria (Reichenbach, mid summer), Scabiosa columbaria, Thymus pulegioides and Heliantemum nummularium (Reichenbach, late summer). The patterns were similar for the out-degree case but, on average, species had a higher in- than out-degree value. In all IPT networks, most of the species (range: 71.4-100\%) donated HP grains to at least one species. Approximately $62 \%$ of these species donated pollen to one or two other species. The highest out-degree $(=13)$ was found for Daucus carota (Reichenbach, late summer), whereas an in-degree of 10 was found for Lotus corniculatus (Hirschtal, midsummer). The percentage of species that acted as hubdonor or -receptor species varied between communities but, in general, more species were identified as hubdonors than receptors (Table 1). Therefore, no statistically clear correlation was detected between the in- and out-degree values of species in all nine communities (Table 1).

Most floral traits showed no statistically clear correlation with either the in- and out-degree (Additional file 1-1). The only statistically clear associations were a negative correlation of the out-degree with stamen length $(z=-2.031, p=0.042)$ and a positive correlation of the out-degree with plant abundance $(z=2.480, p=0.013)$.

Similar patterns were found for the weighted in- and out-degree values (Additional file 1-2). Weighted indegree showed a statistically clear correlation with nectar tube width $(z=2.004, p=0.045)$ and floral abundance $(z=2.344, p=0.019)$ and a positive trend with floral display $(z=1.907, p=0.057)$. Weighted out-degree showed only a statistically clear correlation with floral abundance $(z=5.535, p<0.001)$ and a negative trend for stamen length $(z=-1.859, p=0.063)$ and nectar tube width $(z=2.930, p=0.087)$.

Plant diversity explained more variation in modularity than bee diversity across the nine communities (Fig. 2, Additional file 1-3). The effect of bee species richness (adjusted $R^{2}=0.25^{\text {ns }}$ ) and bee diversity (adjusted $R^{2}=0.06^{\mathrm{ns}}$ ) on modularity was statistically unclear, whereas for bee abundance, we found a statistically clear relationship with modularity (adjusted $R^{2}=0.37^{*}$ ). Modularity showed a statistically clear increase with plant species richness and with flower abundance and diversity of each community (adjusted $R^{2} ; 0.76^{* *}$ (species richness); $0.63^{* * *}$ (flower abundance); $0.89^{* * * *}$ (flower diversity). All three factors were correlated (Pearson's product-moment correlation: $r>0.86^{* * * *}$.

Additionally, floral traits were good predictors for the module composition (randomForest OOB estimate of error rate, mean \pm sd: $7.60 \pm 5.81 \%$, Fig. 3, Table 2, Additional file 1-4). However, those floral traits that had the highest importance for module separation differed between communities (Table 2, Fig. 3). Display size and style length were among the two most important traits for module separation in five out of eight communities. The other traits were among the most important traits in three or four communities.

The percentage of received HP percentage showed no statistically clear correlation with floral traits (Additional file 1-5). However, a statistically clear positive relationship was found with floral abundance $\left(X_{1}^{2}=57.99\right.$, $p<0.001)$ but the explanatory power was low $\left(R_{\text {mar- }}^{2}\right.$ ginal $=0.01, R_{\text {conditional }}^{2}=0.65$ ).

\section{Discussion}

In contrast to flower-visitor interaction networks [22, $25,29]$, the outcome of these interactions, namely pollen transfer, and its association with floral traits has only been investigated in a few communities [17, 26, 27]. Our results show that modularity is well-explained by floral traits but not the quantity and intensity of HP reception or donation. Additionally, network structure is correlated with plant diversity, whereas bee diversity is indicated as being less important. Therefore, we suggest that the topological structure in pollen transfer networks can be explained by the measured floral traits, whereas the intensity or degree of HP transfer is mediated by other factors potentially including various floral traits.

HP receipt and donation (i.e. in- and out-degree) and its intensity (i.e. weighted in- and out-degree) were poorly explained by most of the floral traits. Only the size of the flower entrance (nectar tube width) was positively correlated with weighted in- and out-degree and stamen length negatively with (weighted) out-degree. This indicates that flowers that are easier accessible have a higher quantity of HP and that plant species with short and more hidden stamens, can donate HP to more species [26]. All our communities show an abundance of bees and, particularly, bumble bees, which regularly visit complex flowers, e.g. Lamiaceae, or flowers with deep nectar tubes that are not accessible to many other floral visitors [22]. These plants might have received a large number of visits by bees resulting in an increased out-degree value. This potential effect of abundance and flower-visitor community is in congruence with previous results that have indicated non-consistent out-degrees between communities [30]. Additionally, floral abundance had a positive effect on weighted in-degree and (weighted) out-degree numbers. Therefore, we suggest that, in communities dominated by generalist bees (in our case only 10-20\% specialist species), pollen donation is a function of floral abundance or flower-visitor community rather than of species-specific floral traits.

The modularity of the sampled communities was better predicted by flower abundance and diversity than by those of bees. An increasing flower diversity often results 

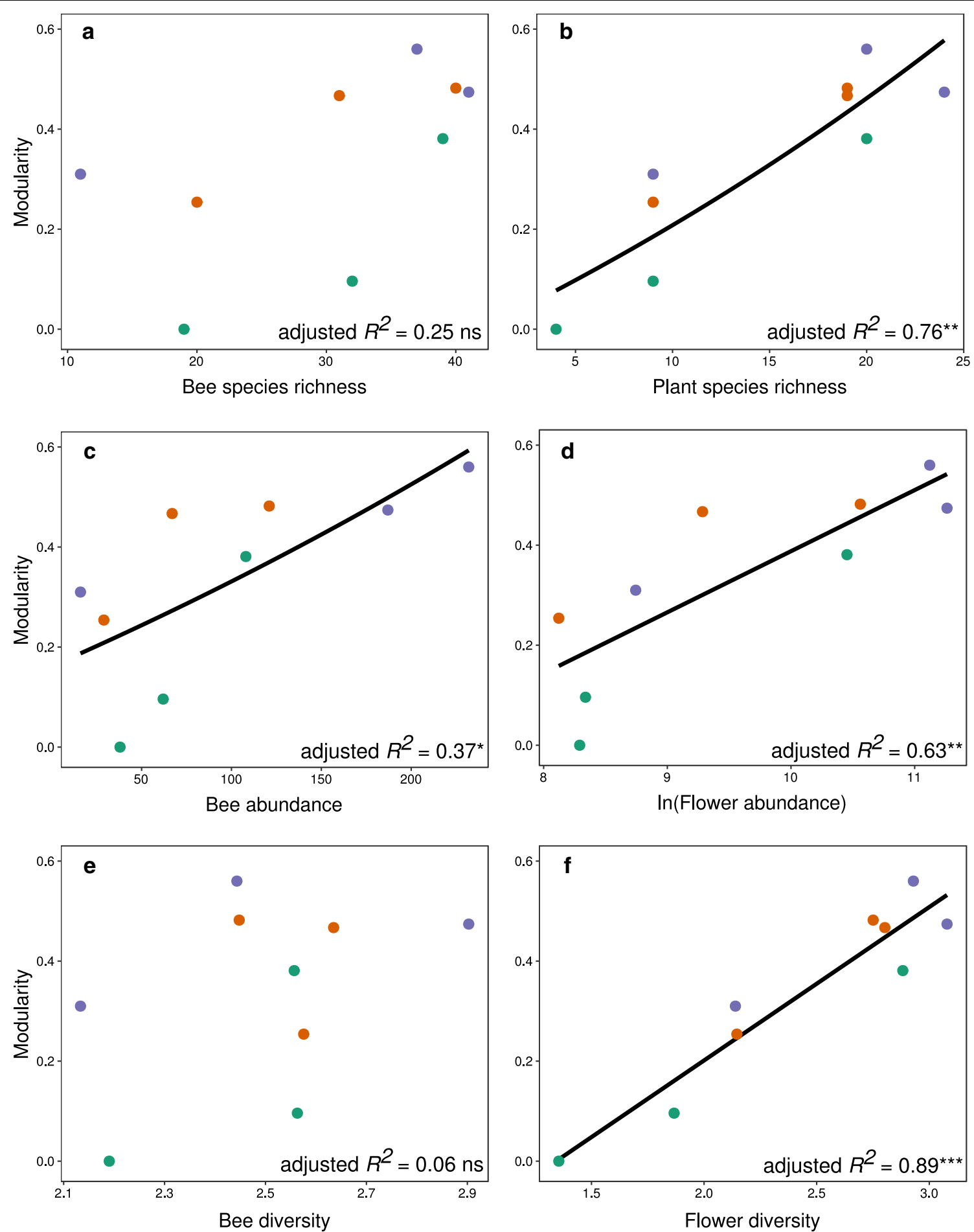

Fig. 2 Correlation between the modularity of the pollen transfer plant-plant network and community parameters. Community parameters are: (a) bee species richness, (b) bee abundance, (c) bee diversity, (d) plant species richness, (e) flower abundance (In-transformed) and (f) flower diversity. Diversity was measured as the Shannon-Wiener index. Different colours represent different locations: Eselsburger Tal (green), Hirschtal (orange), Reichenbach (purple). Each location was sampled once in early, mid and late summer. Black lines are lines of best fit derived from linear models. Adjusted $R^{2}$ are given in the lower right-hand corner of each graph (full results shown in Additional file $1-4$ ). ns $=$ non-significant, ${ }^{*} p<0.05$, ${ }^{* *} p<0.01,{ }^{* * *} p<0.001$ 


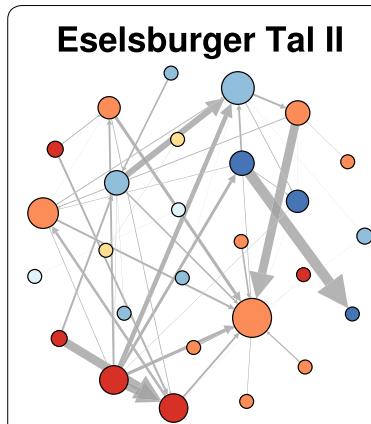

OOB error rate: $4.27 \%$

Three most important traits: stye length (18.19)

inflorescence diameter (17.45)

display size (16.50)

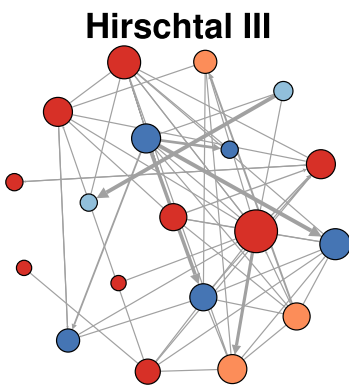

OOB error rate: $6.90 \%$

Three most important traits:

diameter inflorescence (14.12)

display size (11.99)

stamen length (11.08)

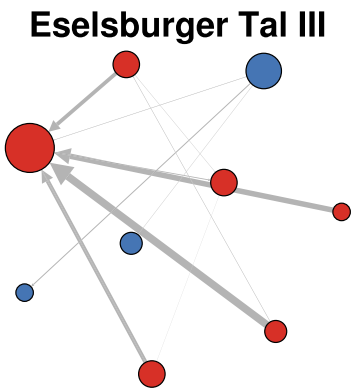

OOB error rate: $0.00 \%$ Three most important traits: stamen length (4.94) nectar tube width (3.92) display size (3.74)

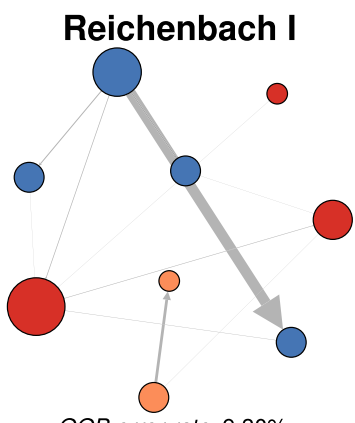

OOB error rate: $9.30 \%$ Three most important traits: nectar tube depth (6.67)

style length (6.45) display size (4.74)

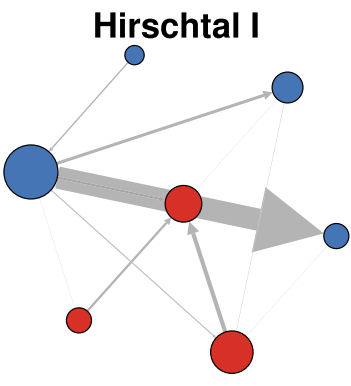

OOB error rate: $2.86 \%$ Three most important traits: nectar tube depth (3.88) style length (3.87) nectar tube width (2.81)

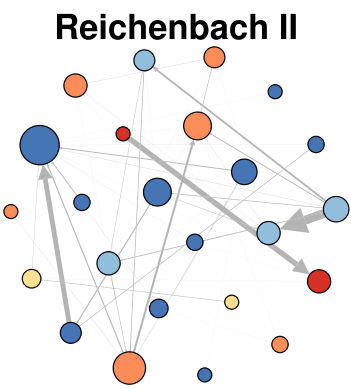

OOB error rate: $13.08 \%$ Three most important traits: style length (17.95) stamen length (14.11) nectar tube depth (13.64)

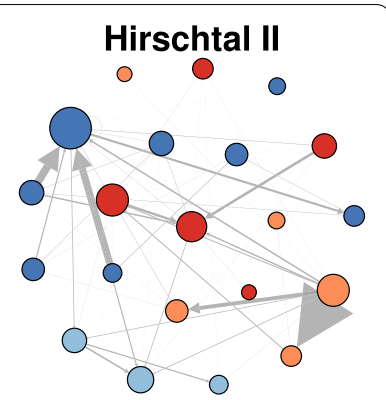

$O O B$ error rate: $18.07 \%$ Three most important traits: style length (11.62) nectar tube width (10.92) stamen length (9.84)

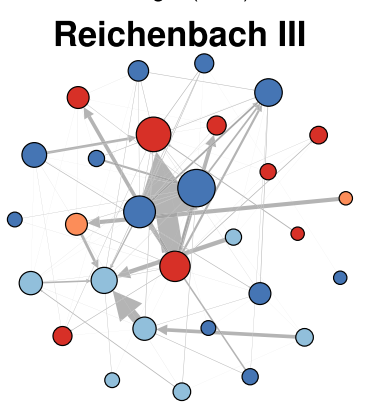

OOB error rate: $6.3 \%{ }^{*}$ Three most important traits: display size (17.36)*

inflorescence diameter $(16.40)^{*}$ nectar tube width $(15.36)^{*}$

Fig. 3 Plant-plant networks based on pollen transfer patterns of eight sampled grassland communities. Each node represents one plant species and each link represents HP transfer between two species. Direction of arrows indicates HP transfer from donor to receptor species and width of arrows indicates the number of transferred pollen grains. Nodes with the same colour are in the same module within one community. Numbers in community names show sampling date: $I=$ early summer, $I=$ mid-summer, $I I=$ late summer. Eselsburger Tal I is not shown as no modules were detected. OOB: the estimate of error rate from random forest analysis shows the percentage of misclassifications, i.e. assignment to the wrong module. The three most important variables for the module classification are given for each community (Table 2). The full results of the random forest analysis are given in Additional file 1-5. For RB III, two solutions of the modularity algorithm (Blondel et al. 2008) are shown as both were equally likely: solution one with four modules / solution two with five modules because one module was split into two plant-plant networks (computed in Gephi version 0.9.2 [41] by using the Fruchterman Reingold layout)

Table 2 Importance of measured floral traits for module separation based on random forest analysis

\begin{tabular}{|c|c|c|c|c|c|c|c|c|}
\hline Floral trait & EB II & EB III & HT I & HT II & HT III & RB I & RB II & RB III \\
\hline Stamen length & 13.76 & 4.94 & 1.58 & 9.84 & 11.08 & 2.94 & 14.11 & $12.01 / 13.77$ \\
\hline Inflor. diameter & 17.45 & 2.52 & 2.40 & 7.53 & 14.12 & 2.87 & 10.86 & $16.40 / 18.35$ \\
\hline Nectar tube depth & 10.38 & 1.27 & 3.88 & 8.01 & 7.11 & 6.67 & 13.64 & $11.88 / 13.01$ \\
\hline Nectar tube width & 13.28 & 3.93 & 2.81 & 10.93 & 5.06 & 3.12 & 9.11 & $15.36 / 15.66$ \\
\hline Display size & 16.50 & 3.73 & 2.12 & 9.32 & 11.99 & 4.74 & 13.63 & $17.36 / 18.36$ \\
\hline Style length & 18.19 & 2.29 & 3.87 & 11.62 & 8.96 & 6.45 & 17.94 & $12.83 / 15.96$ \\
\hline
\end{tabular}

Larger numbers indicate greater importance for module classification. Inflor. diameter =Inflorescence diameter. Location: Eselsburger Tal (EB), Hirschtal (HT), Reichenbach (RB). Sampling date: early summer (I), mid-summer (II), late summer (III). For RB III, two solutions of the modularity algorithm [43] are shown as both were equally likely: solution one with four modules / solution two with five modules because one module was split into two. Eselsburger Tal I is not shown as no modules were detected 
in a higher specialization and higher resource partitioning of pollinator species [16]. This higher specialization limits the number of visited plants and thus might limit the HP transfer between multiple species. Further, in the sampled communities, hyper-generalized honeybees, whose presence increases the interconnectedness of plant species [26], are not ubiquitously found [pers. observ.]. Additionally, the low explanatory power of bee diversity for modularity indicates that other flower-visitor species such as syrphid flies need to be included in the measurements. For bees, only the factor abundance is positively correlated with modularity. Therefore, we suggest that, in our communities, the number of bees rather than the species richness increases resource partitioning and, thus, potentially increases modularity.

We have found that module affiliation is well-explained by floral traits. This indicates that plant species that share the same visitors receive and donate pollen from each other [27]. However, the intensity and quantity of pollen reception and donation is not well explained by measured traits. Therefore, we suggest that the measured traits explain the qualitative part of the interactions (interaction/no interaction) but not the quantitative part. Similarly, pollinator moves between plant species have also been shown to explain the plant species from which HP is received, but not the number of HP grains received [7].

Further, traits that are most important for the separation of plant modules differ between season and site. Even in communities with overlapping plant species, trait importance differs and, thus, none of our measured traits provides a general explanation for interspecific separation. Only display size and style length seem to be more important than other measured traits. The first is related to the width of the flower entrance and, therefore, the accessibility of pollen for the various flower visitors. The second indicates that style position affects HP transfer. Other traits, with lower importance, such as stamen length or nectar tube depth also indicate easier access of the flower visitor to pollen or might be an indicator of different floral morphology. These traits have also been found to be important for pollen transfer patterns in communities in Hawai'i and in high mountain areas [5, $27,31]$. Hence, we suggest that floral traits are important for attraction/efficiency but only some traits (and potentially different ones) are crucial for pollen transfer patterns, which might also depend on the composition of the flower-visitor community.

We found no correlation between floral traits and received HP reception but a positive correlation with floral abundance. This partly contradicts previous studies that have found no correlation with floral traits or that have shown that the \%HP received is influenced by species properties such as floral symmetry, corolla openness and degree of style exsertion [1,26, 27]. Additionally, the $\mathrm{HP} / \mathrm{CP}$ ratio can vary between different species and years and thus suggests an impact of climatic factors [4]. We suggest that these differences between our findings and previous results can be explained by differences in the sampled communities. Whereas the results of Ashman and Arceo-Gómez [1] are based on a meta-analysis, Fang and Huang [27] and Fang et al. [4] have explored subalpine communities that differ in pollinator composition. This indicates that other factors in our planar and kollin meadow communities are more important than in other communities.

Additionally, the present associations between floral traits and HP reception might only become apparent when accounting for differences in flower-visitor interaction frequency. As the number of interactions and the identity of the flower visitors can affect the transported pollen, it can also influence HP reception [11, 32, 33]. However, flower-visitor interaction patterns do not necessarily match HP pollen transfers $[7,10,11]$ and stigmatic pollen loads are consistent across years [4] despite the potentially large variation in interactions between years. These two points suggest that additional plantbased factors, such as floral traits, are important for determining stigmatic heterospecific pollen loads. Further, visitation frequency is often a poor proxy for efficient pollination, i.e. single pollen deposition [34]. Single visit pollen deposition and visitation frequency provide important information on the relative contribution of different taxa visiting a plant species but might be limited when focussing on broader community questions about pollination outcomes and patterns, stigmatic pollen load or seed set [35]. Therefore, the incorporation of interaction frequency and the pollen deposition of different animal species will provide an additional insight and a more mechanistic understanding for the general pollen transfer patterns found here.

As discussed above, floral traits are not or only partly correlated with the intensity or degree of HP transfer [1, $3]$. Thus, we speculate that only a subset of floral traits such as stigma size or position influence the HP transfer/ reception of plants. Fang et al. [4] have suggested that plants deal with HP either by tolerating it or by avoiding it. Therefore, we propose that HP reception also serves as a separate selection pressure for floral traits. To date, pollinator-mediated selection is usually separated into pollinator attraction and efficiency, whereby traits such as nectar tube depth are used in terms of pollinator efficiency [36]. For plants, traits relevant for pollinator attraction/efficiency and HP reception should presumably be separated, i.e. an adaption to one selection pressure might not influence the other, whereas some traits such as floral display size or stigma position may 
influence both pollinator attraction/efficiency and HP reception. Therefore, future studies that aim at disentangling the role of various floral traits during the various stages of pollination and the potential for independent adaption might shed light on the multi-selection pressures on floral traits.

\section{Conclusion}

The results presented here based on the pollen-transfer networks of nine spatially and temporally separated meadow communities in Southern Germany show that our set of floral traits are not well correlated with the quantity of pollen transfer but rather with its quality. We have detected a high impact of floral abundance on network properties, a finding that highlights the importance of community-specific properties and suggests geographic differences. In general, our results indicate that floral traits, known to mediate flower-visitor interactions, can elucidate who is sharing pollen with whom but does not give information about the intensity and quantity of HP transfer, which more likely depends on other floral traits and the identity of flower visitors.

\section{Material and methods}

\section{Study sites}

Our study was conducted between May and July 2018 in three protected dry nutrient-poor limestone grasslands (juniper heathlands), namely Eselsburger Tal $\quad\left(48^{\circ} 36^{\prime} 13.2408^{\prime \prime} \mathrm{N} \quad 10^{\circ} 10^{\prime} 39.6768^{\prime \prime O}\right)$, Hirschtal $\left(48^{\circ} 41^{\prime} 44.1204^{\prime \prime} \mathrm{N} 10^{\circ} 2^{\prime} 8.6388^{\prime \prime O}\right)$ and Reichenbach (Haarberg/Wasserberg; $\left.\quad 48^{\circ} 37^{\prime} 27.2316^{\prime \prime} \mathrm{N} \quad 9^{\circ} 43^{\prime} 59.3796^{\prime \prime} \mathrm{O}\right)$, located on the Swabian Alb, Germany. In each grassland, we used three plots $(50 \times 50 \mathrm{~m})$ established by the BienABest project (https://www.bienabest.de/) and conducted three sampling rounds (early summer, midsummer and late summer): Eselsburger Tal 11-May, 12-July and 30-July; Hirschtal 22-May, 2-July and 25-July; Reichenbach 18-May, 9-July and 24-July-2018. Sampling was conducted on sunny days and restricted to weekdays based on permit regulations. Thus, in total, we sampled nine communities at three locations and time points.

\section{Flower abundance and stigma collection}

In the centre of each plot, we established one transect $(2 \mathrm{~m} \times 50 \mathrm{~m})$, identified all flowering plant species therein and counted the number of flowers per species. For plant species with a large number of small flowers, e.g. Apiaceae, we counted all inflorescences and multiplied them with the mean number of flowers from five inflorescences. To measure stigmatic pollen loads accumulated across the full floral life time, we collected five stigmas of senescent flowers of five different individuals per flowering plant species on each plot (median number of stigmas per species and location $=10$ ). Plant individuals were distributed across the full transect length in a maximum distance of $10 \mathrm{~m}$, when possible. Because of restrictions for protected plant species and for plant species for which no senescent flowers were found, our sample completeness of plant species in each location ranged from 78 to 100\% (mean \pm SD: $86.1 \pm 7.3 \%$; Table 1; Additional file 16). Additionally, we collected flowers in anthesis for each species and established a reference pollen collection. Collected stigmas were stored in Eppendorf tubes in a freezer at $-20^{\circ} \mathrm{C}$ until further preparation.

\section{Pollen evaluation}

For the enumeration and determination of stigmatic HP and CP load, we followed commonly used non-genetic methods $[26,27,30,37,38]$. We placed each stigma in a droplet of Alexanders' stain [39] on a microscopic slide, covered each preparation with a coverslip and sealed the preparation with transparent nail varnish under a stereoscopic microscope (Stemi 2000-CS; Carl ZEISS AG). Subsequently, the microscopic slides where incubated $48 \mathrm{~h}$ at $50{ }^{\circ} \mathrm{C}$. Pollen for the reference collection was prepared accordingly. For protected species, our reference collection was supplemented by images found in the open source database PalDat (PalDat, 2000). For the reference collection, we took photographs from pollen grains at various planes and measured pollen size for all species under the light microscope (Scope.A1; Carl ZEISS AG) at 63-100 × magnification.

Pollen was evaluated under the same light microscope. All CP and HP were enumerated and determined by using the prepared reference pollen collection. In cases in which the identification of HP origin was not possible from our reference collection, we categorized the pollen as "unknown" (unidentified pollen was found on 9\% of all stigmas and accounted for $10 \%$ of all sampled HP grains). If the identification of pollen was unclear between two species, we assumed the species with the higher abundance as being the origin.

\section{Floral trait measurements}

For each plant species (total $n=52$ ), we collected five individuals per species from one or two locations. For five species, we only collected three or four individuals. Plant species under protection were not collected (Additional file 1-6). We measured seven morphological floral traits known to mediate plant-pollinator interaction $[15,21,23,25]$ by using a caliper rule under a stereomicroscope (Stemi 2000-CS; Carl ZEISS AG): (1) stamen length $[\mathrm{mm}],(2)$ inflorescence diameter $[\mathrm{mm}]$, (3) nectar tube width [mm], (4) nectar tube depth [mm] (both zero if no nectar tube was present), (5) display size of flower 
[mm] (maximum distance between apical ends of two petals) and (6) style length [mm].

\section{Bee species richness and abundance}

Data on bee species and abundance were provided by the BienABest project (www.bienabest.de). Here, at each location $(n=3)$, each plot $(n=3)$ and each sampling date $(n=3)$, wild bees were sampled during one day once before and once after noon by means of walking along the available resources for $25 \mathrm{~min}$ [40]. Each bee observed was noted and the bees that could not be identified to species level at first sight were caught and identified in the laboratory. This sampling procedure allowed us to determine differences in bee abundance between sites but may not have represented accurate absolute numbers of bees per site. For example, one day per week (or even fewer) is often used in monitoring programmes when determining abundance differences between sites or years [41-43]. Further, if the flower longevity in our grasslands is 1 to $11 / 2$ weeks, an estimation of the abundance at one particular time should provide an adequate approximation of abundance within this timeframe.

\section{Statistical analysis}

For analysing the pollen transfer based on stigmatic HP loads within the different communities (all three plots were summarized), we created unipartite directed networks by using the open-source software Gephi version 0.9.2 [44]. Each established link between two plants was defined as pollen grains from one plant species (a) found on the stigma of another plant species (b). Each link was weighted by the number of pollen grains received by the receptor species (b) from the donor species (a). In each network, we calculated the in- and out-degree number for each species. The in-degree was defined as the number of incoming links, whereas the out-degree was defined as the number of outgoing links. Based on the in- and out-degree values, we identified whether the plant species act as a hub-donor (in-degree $<$ out-degree), a hub-receptor (in-degree $>$ out-degree) or a neutral species (in-degree $=$ out-degree) [45]. Further, the weighted in- and out-degree values were calculated [44], which weighted each link by its intensity, i.e. pollen grain number. Lastly, the modularity was calculated using the optimization algorithm of Blondel et al. [46]. We used a resolution factor of 0.7 [47] and weighted links according the amount of HP. As modularity and species composition in each module can vary between runs, the algorithm was run 25 times for each network. For EBI, HTI and HTII, all runs resulted in the same number of modules, modularity and species composition in each module. For EBII and EBIII, 20 runs resulted in the same results and, for RBI and RBII, 22 and 23 runs. For all these networks, we considered the results that appeared $>80 \%$ as the most likely ones. For RBIII, two solutions were equally likely, both appearing 11 times. Both had a similar modularity of 0.474 and 0.472 . However, they differed in the module assignment of three species (Table 2). Analyses were calculated with both. All parameters of plant-plant networks and graphs were carried out in Gephi version 0.9.2 [44].

Further statistical analyses were performed in $R$ version 3.4.4 [48]. To test the relationship between in- and out-degree values and measured floral traits and flower abundance (question I), we performed generalized linear mixed models (GLMMs) with a beta distribution. The mean stamen length, inflorescence diameter, nectar tube depth, nectar tube width, display size, style length or flower abundance were included as the fixed factor and the community and plant species as the random factor. Flower abundance was $\ln$-transformed to reduce the importance of highly abundant small flowers, e.g. Apiaceae. For standardization, in- and out-degree values were divided by the number of flowering plant species per community and transformed according to CribariNeto \& Zeileis [49] for beta regression. All models were calculated using the glmmTMB-package [50] with the $\operatorname{glmmTMB}$-function. To test the relationship between weighted in- and out-degree values and measured floral traits and flower abundance (question I), we performed Poisson-distributed GLMMs by using the lme4-package [51] with the glmer-function. The same model structure as describe for in- and out-degree values was used. The fit for each model was validated using the DHARMa-package [52] as described in the package vignette.

To test whether the modularity of plant-plant networks was affected by community parameters, i.e. bee species richness, abundance, diversity, plant species richness, flower abundance (ln-transformed) and diversity (question II), we used linear regression models (LM). Bee and flower diversities were calculated as Shannon-Wiener indices by using the diversity-function of the vegan-package [53]. If necessary, modularity was $\ln +1$-transformed to achieve normality.

To address whether floral traits could explain the module classification of the plant species (question III), we used random forest analysis by using the randomForest-package [54]. Random forest is a machine learning algorithm [55]; here, it assigned plant individuals to the predefined modules in multiple iterations based on the measured floral traits and estimated the importance of each floral trait for the correct classification of each plant individual. For this analysis, we used ntree $=10,000$ bootstrap replicates drawn with the $m$ try $=2$ variable randomly selected at each node. To identify the most important variable for classification, we used the importance-function of the same package. 
To test whether floral traits and floral abundance are linked to the ratio of the received HP percentage (question IV), we performed binomial GLMMs, as the beta distribution showed a poor model fit. All models were calculated using the lme4-package [51] with the glmerfunction. The significance for each fixed factor was determined via the likelihood ratio test by comparing the model including the fixed and random factors with the model only including random factors. If the model convergence failed when we used the default arguments, we first reran the model by restarting from the previous fit, this increased the maximum number of iterations. In those cases in which the restart did not result in model convergence, we replaced the default optimizer in the second phase with the bobyqa-optimizer. This step always resulted in model convergence. The relative contribution of the model factors to the variation in the interaction patterns was estimated by calculating $R_{\text {marginal }}^{2}$ and $R_{\text {con- }}^{2}$ ditional [56]. Each model was validated as described above.

To estimate sample completeness, we calculated sample and incidence-based rarefaction curves for each community by using the iNEXT-package [57]. Overall, for most species, curves did not reach an asymptote and curves for the same species differed between communities. However, within-community extrapolated curves showed no changes in the relative positions of the various species compared with interpolated curves. Further, none of the overlapping curves indicated that the relative position of the species to each other would have been stable with further sampling. As the sampling was the same in all communities, the absolute values might be biased, whereas the relative values between communities should not be affected, e.g. the absolute values of modularity compared with correlations of modularity having community properties. Therefore, the results can be interpreted accordingly within our study. Full results are shown in Additional file 1-7.

\section{Supplementary information}

Supplementary information accompanies this paper at https://doi. org/10.1186/s12898-020-00323-5.

Additional file 1. 1. Correlation between floral traits and in- and outdegree. 2. Correlation between floral traits and weighted in- and outdegree. 3. Correlations between modularity and community parameters. 4. Results of random forest analysis for modularity and floral traits. 5. Correlations between proportion of HP and floral traits. $\mathbf{6}$. Flower abundance and plant species sampled. 7. Rarefaction curves for each community.

\section{Acknowledgements}

We are grateful to Sandra Voxbrunner for collecting pollen samples. We thank H. R. Schwenninger who was substantially involved in the setting up and running of the BienABest project and in bee sampling and determination and L. Woppowa and H. Seitz for coordination of the BienABest project. We thank the Regierungspräsidium Stuttgart, Germany for giving permission to collect flower and bee samples in these areas. Additionally, we thank two anonymous reviewers for valuable comments on earlier versions of this paper. Open Access funding was partially provided by Ulm University under the DEAL-agreement.

\section{Authors' contributions}

JK conceived the study. JK designed the study with input from MA. MP and JK collected and analysed pollen samples and floral traits. MA, HB and UN designed the bee sampling protocol. HB, SK and UN collected and determined the bee specimens. MP and JK performed the statistical analysis. JK wrote the first version of the manuscript. All authors contributed to the final version. All authors read and approved the final manuscript.

\section{Funding}

Open Access funding enabled and organized by Projekt DEAL. The BienABest project is funded within the Federal Programme for Biological Diversity by the Federal Agency for Nature Conservation with resources from the Federal Ministry for the Environment, Nature Conservation and Nuclear Safety. The project is financially supported by the Ministry of the Environment, Climate Protection and the Energy Sector Baden-Württemberg, BASF SE and the Bee Care Centre of Bayer AG.

\section{Availability of data and materials}

The data used in this paper are available on Figshare https://doi.org/10.6084/ m9.figshare.13013282.

\section{Ethics approval and consent to participate}

The sampling of plants and bees was approved and conducted in accordance with the regulations of the regional council (Regierungspräsidium) Stuttgart, Baden-Wuerttemberg, Germany (permit number/Aktenzeichen: plants 55-8841.06/14/UniUImKuppler; bees 55-8850.68/14/UniUlm).

\section{Consent for publication}

Not applicable

\section{Competing interest}

The authors declare that they have no competing interests.

Received: 23 April 2020 Accepted: 23 September 2020

Published online: 06 October 2020

\section{References}

1. Ashman TL, Arceo-Gómez G. Toward a predictive understanding of the fitness costs of heterospecific pollen receipt and its importance in coflowering communities. Am J Bot. 2013;100:1061-70.

2. Arceo-Gómez G, Alonso C, Ashman TL, Parra-Tabla V. Variation in sampling effort affects the observed richness of plant-plant interactions via heterospecific pollen transfer: Implications for interpretation of pollen transfer networks. Am J Bot. 2018;105:1601-8.

3. Arceo-Gómez G, Schroeder A, Albor C, Ashman TL, Knight TM, Bennett $J M$, et al. Global geographic patterns of heterospecific pollen receipt help uncover potential ecological and evolutionary impacts across plant communities worldwide. Sci Rep. 2019;9:1-9.

4. Fang Q, Gao J, Armbruster WS, Huang S-Q. Multi-year stigmatic pollenload sampling reveals temporal stability in interspecific pollination of flowers in a subalpine meadow. Oikos. 2019;00:1-9.

5. Huang ZH, Le LH, Huang SQ. Interspecific pollen transfer between two coflowering species was minimized by bumblebee fidelity and differential pollen placement on the bumblebee body. J Plant Ecol. 2015;8:109-15.

6. Arceo-Gómez G, Ashman TL. Heterospecific pollen deposition: Does diversity alter the consequences? New Phytol. 2011;192:738-46.

7. Fang Q, Huang SQ. A paradoxical mismatch between interspecific pollinator moves and heterospecific pollen receipt in a natural community. Ecology. 2016;97:1970-8.

8. Linder HP, Midgley J. Anemophilous plants select pollen from their own species from the air. Oecologia. 1996;108:85-7.

9. Minnaar C, Anderson B, De Jager ML, Karron JD. Plant-pollinator interactions along the pathway to paternity. Ann Bot. 2019;123:225-45. 
10. Ballantyne G, Baldock KCR, Willmer PG. Constructing more informative plantpollinator networks: Visitation and pollen deposition networks in a heathland plant community. Proc R Soc B Biol Sci. 2015;282:20151130.

11. Ballantyne $G$, Baldock KCR, Rendell L, Willmer PG. Pollinator importance networks illustrate the crucial value of bees in a highly speciose plant community. Sci Rep. 2017;7:1-13.

12. Jordano P, Bascompte J, Olesen JM. Invariant properties in coevolutionary networks of plant-animal interactions. Ecol Lett. 2003;6:69-81.

13. Blüthgen N. Why network analysis is often disconnected from community ecology: A critique and an ecologist's guide. Basic Appl Ecol. 2010;11:185-95.

14. Vázquez DP, Chacoff NP, Cagnolo L. Evaluating multiple determinants of the structure of plant-animal mutualistic networks. Ecology. 2009;90:2039-46.

15. Junker RR, Blüthgen N, Brehm T, Binkenstein J, Paulus J, Martin Schaefer $\mathrm{H}$, et al. Specialization on traits as basis for the niche-breadth of flower visitors and as structuring mechanism of ecological networks. Funct Ecol. 2013;27:329-41.

16. Fründ J, Linsenmair KE, Blüthgen N. Pollinator diversity and specialization in relation to flower diversity. Oikos. 2010;119:1581-90.

17. Arceo-Gómez G, Abdala-Roberts L, Jankowiak A, Kohler C, Meindl GA, Navarro-Fernández CM, et al. Patterns of among- and within-species variation in heterospecific pollen receipt: The importance of ecological generalization. Am J Bot. 2016;103:396-407.

18. Ebeling A, Klein AM, Tscharntke T. Plant-flower visitor interaction webs: Temporal stability and pollinator specialization increases along an experimental plant diversity gradient. Basic Appl Ecol. 2011;12:300-9. https://doi. org/10.1016/j.baae.2011.04.005.

19. Olesen JM, Bascompte J, Dupont $Y L$, Jordano P. The modularity of pollination networks. Proc Natl Acad Sci. 2007;104:19891-6.

20. Kratochwil A, Beil M, Schwabe A. Complex structure of pollinator-plant interaction-webs: random, nested, with gradients or modules? Apidologie. 2009;40:634-50.

21. Junker RR, Parachnowitsch AL. Working towards a holistic view on flower traits - How floral scents mediate plant-animal interactions in concert with other floral characters. J Indian Inst Sci. 2015;95:1-26.

22. Stang M, Klinkhamer PGL, Van Der Meijden E. Size constraints and flower abundance determine the number of interactions in a plant-flower visitor web. Oikos. 2006;112:111-21.

23. Kuppler J, Höfers MK, Wiesmann L, Junker RR. Time-invariant differences between plant individuals in interactions with arthropods correlate with intraspecific variation in plant phenology, morphology and floral scent. New Phytol. 2016;210:1357-68.

24. Burger $H$, Dötterl $S$, Ayasse M. Host-plant finding and recognition by visual and olfactory floral cues in an oligolectic bee. Funct Ecol. 2010;24:1234-40.

25. Fenster CB, Armbruster WS, Wilson P, Dudash MR, Thomson JD. Pollination syndromes and floral specialization. Annu Rev Ecol Evol Syst. 2004:35:375-403.

26. Johnson AL, Ashman TL. Consequences of invasion for pollen transfer and pollination revealed in a tropical island ecosystem. New Phytol. 2019;221:142-54.

27. Fang Q, Huang SQ. A directed network analysis of heterospecific pollen transfer in a biodiverse community. Ecology. 2013;94:1176-85.

28. Arceo-Gómez G, Alonso C, Abdala-Roberts L, Parra-Tabla V. Patterns and sources of variation in pollen deposition and pollen tube formation in flowers of the endemic monoecious shrub Cnidoscolus souzae (Euphorbiaceae). Plant Biol. 2016;18:594-600.

29. Junker RR, Blüthgen N, Keller A. Functional and phylogenetic diversities of plant communities differently affect the structure of flower-visitor interactions and reveal convergences in floral traits. Evol Ecol. 2015;29:437-50.

30. Tur C, Sáez A, Traveset A, Aizen MA. Evaluating the effects of pollinatormediated interactions using pollen transfer networks: Evidence of widespread facilitation in south Andean plant communities. Ecol Lett. 2016;19:576-86

31. Harder LD, Johnson SD. Darwin's beautiful contrivances: evolutionary and functional evidence for floral adaptation. New Phytol. 2009;183:530-45.

32. Gyan KY, Woddell SRJ. Analysis of insect pollen loads and pollination efficiency of some common insect visitors of four species of woody Rosaceae. Funct Ecol. 1987;1:269-74.

33. Alarcón R. Congruence between visitation and pollen-transport networks in a California plant-pollinator community. Oikos. 2010;119:35-44.
34. King C, Ballantyne G, Willmer PG. Why flower visitation is a poor proxy for pollination: Measuring single-visit pollen deposition, with implications for pollination networks and conservation. Methods Ecol Evol. 2013;4:811-8.

35. Willcox BK, Aizen MA, Cunningham SA, Mayfield MM, Rader R. Deconstructing pollinator community effectiveness. Curr Opin Insect Sci. 2017;21:98104. https://doi.org/10.1016/j.cois.2017.05.012.

36. Caruso CM, Eisen KE, Martin RA, Sletvold N. A meta-analysis of the agents of selection on floral traits. Evolution. 2019;73:4-14.

37. Arceo-Gómez G, Jameel Ml, Ashman TL. Effects of heterospecific pollen from a wind-pollinated and pesticide-treated plant on reproductive success of an insect-pollinated species. Am J Bot. 2018;105:836-41.

38. Arceo-Gómez G, Kaczorowski RL, Patel C, Ashman TL. Interactive effects between donor and recipient species mediate fitness costs of heterospecific pollen receipt in a co-flowering community. Oecologia. 2019;189:1041-7.

39. Dafni A, Pacini E, Nepi M. Pollen and stigma biology. In: Dafni A, Kevan PG, Husband BC, editors. Practical pollination biology. Cambridge: Environquest Ltd; 2005. p. 83-142.

40. Neumüller $U$, Burger $H$, Krausch S, Blüthgen $N$, Ayasse M. Interactions of local habitat type, landscape composition and flower availability moderate wild bee communities. Landsc Ecol. 2020. https://doi.org/10.1007/s1098 0-020-01096-4

41. Kaiser-Bunbury CN, Muff S, Memmott J, Müller CB, Caflisch A. The robustness of pollination networks to the loss of species and interactions: A quantitative approach incorporating pollinator behaviour. Ecol Lett. 2010;13:442-52.

42. Chacoff NP, Resasco J, Vázquez DP. Interaction frequency, network position, and the temporal persistence of interactions in a plant-pollinator network. Ecology. 2018;99:21-8.

43. Seibold S, Gossner MM, Simons NK, Blüthgen N, Müller J, Ambarlı D, et al. Arthropod decline in grasslands and forests is associated with landscapelevel drivers. Nature. 2019;574:671-4.

44. Bastian M, Heymann S, Jacomy M. Gephi: an open source software for exploring and manipulating networks. International AAAI Conference on Weblogs and Social Media. 2009. www.gephi.org. Accessed 6 Jan 2019.

45. Newman M. The structure and function of complex networks. Siam Rev. 2003;45:167-256.

46. Blondel VD, Guillaume JL, Lambiotte R, Lefebvre E. Fast unfolding of communities in large networks. J Stat Mech Theory Exp. 2008. https://doi. org/10.1088/1742-5468/2008/10/P10008

47. Arceo-Gómez G, Kaczorowski RL, Ashman T-L. A network approach to understanding patterns of coflowering in diverse communities. Int J Plant Sci. 2018;179:569-85.

48. R CoreTeam. R: a language and environment for statistical computing. Version 3.4.4. 2018. https://www.r-project.org/. Accessed 29 Feb 2020.

49. Cribari-Neto F, Zeileis A. Beta regression in R. J Stat Softw. 2010;34:1-24.

50. Brooks ME, Kristensen K, van Benthem KJ, Magnusson A, Berg CW, Nielsen A, et al. glmmTMB balances speed and flexibility among packages for zeroinflated generalized linear mixed modeling. R J. 2017;9:378-400.

51. Pinheiro J, Bates D, DebRoy S, Sarkar D, Team RC. nlme: Linear and Nonlinear Mixed Effects Models. R Package version 31-137. 2018. https://CRAN.R-proje ct.org/package =nlme. Accessed 29 Feb 2020

52. Hartig F. DHARMa: Residual diagnostics for hierarchical (multi-level/mixed) regression models. R package version 0.2.6. 2020. https://cran.r-project.org/ package=DHARMa. Accessed 29 Feb 2020.

53. Oksanen J, Blanchet FG, Friendly M, Kindt R, Legendre P, McGlinn D, et al. Vegan: Community Ecology Package. R package version 2.5-5. 2019. https:// cran.r-project.org/package=vegan. Accessed 29 Feb 2020.

54. Liaw A, Wiener M. Classification and regression by randomForest. R News. 2002;2:28-2.

55. Breiman L. Random Forests. Mach Learn. 2001;45:5-32.

56. Nakagawa $\mathrm{S}$, Schielzeth $\mathrm{H}$. A general and simple method for obtaining R2 from generalized linear mixed-effects models. Methods Ecol Evol. 2013;4:133-42.

57. Chao A, Gotelli NJ, Hsieh TC, Sander EL, Ma KH, Colwell RK, et al. Rarefaction and extrapolation with Hill numbers: A framework for sampling and estimation in species diversity studies. Ecol Monogr. 2014;84:45-67.

\section{Publisher's Note}

Springer Nature remains neutral with regard to jurisdictional claims in published maps and institutional affiliations. 